Claremont Colleges

Scholarship@ Claremont

WM Keck Science Faculty Papers

W.M. Keck Science Department

8-10-1992

\title{
Geometrical Phases and Symmetries in Dissipative Systems
}

\author{
Adam S. Landsberg \\ Claremont McKenna College; Pitzer College; Scripps College
}

\section{Recommended Citation}

Landsberg, A.S. "Geometrical Phases and Symmetries in Dissipative Systems." Physical Review Letters 6.6 (1992): 865.

This Article is brought to you for free and open access by the W.M. Keck Science Department at Scholarship @ Claremont. It has been accepted for inclusion in WM Keck Science Faculty Papers by an authorized administrator of Scholarship @ Claremont. For more information, please contact scholarship@cuc.claremont.edu. 


\title{
PHYSICAL REVIEW \\ LETTERS
}

\begin{tabular}{lcc}
\hline \hline Volume 69 & 10 AUGUST 1992 & Number 6 \\
\hline
\end{tabular}

\section{Geometrical Phases and Symmetries in Dissipative Systems}

\author{
A. S. Landsberg \\ Department of Physics, University of California at Berkeley, Berkeley, California 94720 \\ (Received 13 January 1992)
}

\begin{abstract}
A geometrical phase is constructed for dissipative dynamical systems possessing continuous symmetries. It emerges as the natural analog of the holonomy associated with the adiabatic variation of parameters in quantum-mechanical and classical Hamiltonian systems. In continuous media, the physical manifestation of this phase is a spatial shift of a wave pattern, typically a translation or rotation. An illustration associated with pattern formation in fluids is provided.
\end{abstract}

PACS numbers: $03.40 .-t, 03.20 .+\mathrm{i}$

The geometrical phase discovered by Berry [1] has been the focus of much attention in recent years, and a number of efforts have been made to generalize this notion (e.g., [2-4]). Recently, progress has been made in extending some of these ideas to an important class of systems, those described by classical dissipative field theories. In particular, it was shown in [5] that temporal phases of a geometric nature can arise in dissipative systems exhibiting stable oscillations. In this Letter, we consider nonlinear dissipative systems which possess continuous symmetries (e.g., translations, rotations) and show how these spatial symmetries can lead to a new type of geometrical phase shift. The phase shift described here is a very general phenomenon which arises when the control parameters of a system undergo cyclic, adiabatic evolution. This new phase has direct physical implications, which makes it amenable to experimental observation.

We begin with a simple qualitative description of the effect. Consider any dissipative system with a continuous spatial symmetry. One might imagine, for instance, a translationally invariant system consisting of an unbounded layer of viscous fluid. The presence of a continuous symmetry group will have some important physical consequences. First note that for any particular wave pattern exhibited by the system there exists an entire family of "equivalent" patterns generated by the group action (i.e., by arbitrary translations of the original pattern). Patterns may therefore be grouped into equivalence classes, wherein all members of a given equivalence class are related by symmetry. The group action can thus be viewed as inducing motion within a given equivalence class.

The existence of these equivalence classes has important ramifications for the stability of a wave pattern. To see this, consider the effect of a small perturbation on a "stable" wave pattern. In a typical dissipative system which lacks spatial symmetry, it is expected that the perturbation will decay exponentially, with the original stable pattern reemerging. However, in the presence of symmetry, the initial and final patterns need not be identical. Instead, the final pattern need only lie in the same equivalence class as the original, and could differ by a small translation. In other words, perturbations to a wave pattern in the direction defined by the group action do not decay away. The continuous symmetry is therefore said to have generated a "neutrally stable" direction or mode for the system.

Now suppose that one observes a stable wave pattern in an experiment for some fixed value of the control parameters. For simplicity, assume the wave to be stationary. What happens to this pattern if the control parameters of the system are varied in an adiabatic manner? To answer this, we first assume the existence of a whole range of parameter values (encompassing the initial settings) for which stable patterns exist. Each point in parameter space is then associated with a particular stable pattern (or, more precisely, with the equivalence class of that stable pattern). It is assumed that paths through parameter space connect these stable solutions in a smooth manner. (This requirement simply ensures that the initial wave pattern will not undergo a bifurcation when the 
control parameters begin to vary.) This said, we can now understand the evolution of the initial pattern. As the parameters slowly vary, the wave pattern deforms, and a particular path in parameter space is swept out. By assumption, each point on this path is associated with a particular stable pattern. On physical grounds we argue that the observed wave pattern should, at each instant, lie close to the stable pattern associated with that point of parameter space. This can be expected since dissipation will bring about a rapid collapse of the wave pattern onto these associated stable solutions on a time scale fast relative to the adiabatic time scale. Of course, what is really meant here is that the pattern collapses onto some member of the equivalence class of each stable solution. Thus, when the control parameters are returned to their initial settings, the emergent wave pattern will lie in the same equivalence class as the original. It will therefore, in general, be translated from its initial configuration. This translation between the initial and final wave patterns may be understood as a geometrical phase shift. In this Letter we provide a simple derivation for this spatial phase for an arbitrary Abelian symmetry group, and demonstrate that the phase is truly geometrical in nature.

Consider a general nonlinear dissipative system with spatial symmetry. We express the partial differential equations governing this system in the general form

$$
\frac{d \Psi}{d t}(t, x, \lambda)=\mathcal{F}(\lambda) \Psi(t, x, \lambda),
$$

where $\Psi$ is a vector-valued field, and $\mathcal{F}$ a nonlinear, time-independent operator involving spatial coordinates $x \in \Re^{n}$ and control parameters $\lambda \in \Re^{m}$. By assumption, these governing equations admit a one-parameter $(\theta)$ Lie group of transformations $\left\{\Gamma_{\theta}\right\}$, reflecting the underlying symmetry of the physical system. Common examples include the translation group $\mathrm{T}$ and rotations $\mathrm{SO}(2)$. All symmetry groups considered here are assumed to be strictly spatial in nature, independent of time.

The invariance of the equations under the group implies that for any solution $\Psi$ of (1), $\Gamma_{\theta} \Psi$ is also a solution. For linear groups this simply implies that the operators $\mathcal{F}$ and $\Gamma_{\theta}$ commute. The parametrization of the group may be chosen such that

$$
\Gamma_{\theta_{1}} \Gamma_{\theta_{2}}=\Gamma_{\theta_{1}+\theta_{2}}
$$

with $\Gamma_{0}$ the identity. The boundary conditions for (1) will remain unspecified, but are required to respect the symmetry of the problem. The generator of the symmetry group will be denoted by $\chi$. As noted earlier, any solution $\Psi$ is only neutrally stable to perturbations along the direction determined by the group action. This neutral direction is given by $\chi^{\Psi}$.

Now consider a solution of $(1), \psi\left(x, \lambda_{0}\right)$, representing the initial stationary wave pattern observed in the system at parameter setting $\lambda_{0}$, i.e.,

$$
\mathcal{F}\left(\lambda_{0}\right) \psi\left(x, \lambda_{0}\right)=0 \text {. }
$$

Assume this solution to be stable for this parameter value. Since we are precluding the possibility of bifurcations, there will be some region of parameter space for which nearby stable stationary solutions exist. At each fixed parameter setting $\lambda$ we write the corresponding stable solution as $\psi(x, \lambda)$. However, since each of these stable solutions belongs to its own equivalence class, we could equally well have chosen a different member from each class. We thus write the solution as $\Gamma_{g(\lambda)} \psi(x, \lambda)$, where $g$ is an arbitrary function of the parameters $\lambda$. The function $g$ corresponds to a specific choice of gauge.

We now wish to examine the evolution of the initial wave $\psi\left(x, \lambda_{0}\right)$ as the parameters begin to vary adiabatically. Although our formulation will be solely in terms of stationary patterns, with only minor modifications, certain propagating wave forms may also be accommodated through a transformation to a comoving frame. This will be illustrated in an example. We introduce a slow time dependence into the parameters $[\lambda \rightarrow \lambda(\epsilon t)]$, where $\epsilon$ $(\ll 1)$ is a measure of adiabaticity. The subsequent evolution of the system is studied using a multiple time scale analysis of Eq. (1). We set

$$
\tau=\epsilon t, \quad \frac{d}{d t}=\partial_{t}+\epsilon \partial_{\tau}, \quad \Psi=\Psi_{0}+\epsilon \Psi_{1}+\cdots .
$$

At lowest order in this asymptotic expansion we recover an equation for $\Psi_{0}$ identical to (1),

$$
\partial_{t} \Psi_{0}(t, x, \lambda(\tau))=\mathscr{F}(\lambda(\tau)) \Psi_{0}(t, x, \lambda(\tau)) .
$$

At this level, the control parameters $\lambda$ appear "frozen," since they evolve only on the slow time scale $\tau$. Therefore, the stable solution is simply

$$
\Psi_{0}=\Gamma_{g(\lambda)} \psi(x, \lambda) .
$$

For this solution to coincide with the initial pattern $\psi\left(x, \lambda_{0}\right)$ at the starting time, we require $\lambda(0)=\lambda_{0}$ and $g\left(\lambda_{0}\right)=0$. Apart from this, the function $g$ remains arbitrary.

At next order in the expansion we obtain an equation for $\Psi_{1}$ of the form

$$
\partial_{t} \Psi_{1}=D \mathcal{F}\left(\Gamma_{g} \psi\right) \Psi_{1}-\partial_{\tau} \Gamma_{g} \psi,
$$

where $D \mathcal{F}\left(\Gamma_{g} \psi\right)$ denotes the linearization of the operator $\mathcal{F}$ about the lowest-order solution $\Gamma_{g} \psi$. Several remarks are in order. First note that since $\Gamma_{g} \psi$ represents a stable solution in a dissipative system, the spectrum of the operator $D \mathcal{F}\left(\Gamma_{g} \psi\right)$ is constrained to lie entirely in the left half of the complex plane, bounded away from the imaginary axis, save a single isolated zero eigenvalue associated with the direction of the neutral model. This neutral direction is $\chi \Gamma_{g} \psi$. Second, we note that the final term in Eq. (4) may be regarded as a driving or perturbing term for the system. Not all "directions" associated with this perturbation will be relevant, and we may decompose the driving term into resonant and nonresonant pieces. In particular, only the component of the driving term in the direction of the neutral mode will generate secular 
growth. The growth of the other modes remains bounded due to dissipation. This can be seen by defining the operator

$$
\mathscr{E} \equiv-\int_{0}^{t} d s e^{s D \mathcal{F}\left(\Gamma_{g} \psi\right)}
$$

By applying this operator to the driving term, we obtain the formal solution of (4), i.e., $\Psi_{1}=\mathscr{E}_{\tau} \partial_{\tau} \Gamma_{g} \psi$. Now note that $\mathscr{E}$ acting on any stable eigenvector of the linear operator $D \mathcal{F}\left(\Gamma_{g} \psi\right)$ yields only bounded growth, which will prove negligible in the asymptotic limit. However, $\mathscr{E}$ applied to the null vector (i.e., the neutral mode $\chi \Gamma_{g} \psi$ ) of the linear operator produces linear (secular) growth. Thus, on a time scale long compared to the dissipative time scale of the problem but short relative to the adiabatic time scale, the evolution of the system is dominated by this resonant component. One may therefore replace the driving term $\partial_{\tau} \Gamma_{g} \psi$ by its projection onto the null space of $D \mathcal{F}\left(\Gamma_{g} \psi\right)$, i.e.,

$$
\partial_{\tau} \Gamma_{g} \psi \rightarrow \frac{\left(v_{g}, \partial_{\tau} \Gamma_{g} \psi\right)}{\left(v_{g}, \chi \Gamma_{g} \psi\right)} \chi \Gamma_{g} \psi
$$

Here $v_{g}$ is the adjoint null vector satisfying $D \mathcal{F}\left(\Gamma_{g} \psi\right)^{\dagger} v_{g}$ $=0$, and $(\cdot, \cdot)$ denotes an inner product which is invariant (see [6]) under the group action. Applying $\mathscr{E}$ to this projection yields the asymptotic form of $\Psi_{1}$. We find

$$
\epsilon \Psi_{1}=-\epsilon t \frac{\left(v_{g}, \partial_{\tau} \Gamma_{g} \psi\right)}{\left(v_{g}, \chi \Gamma_{g} \psi\right)} \chi \Gamma_{g} \psi
$$

To make the meaning of this expression more transparent, first notice that for times $t \ll 1 / \epsilon$, the term $\epsilon t$ above plays the role of a differential $d \tau$. We can thus reexpress $\epsilon \Psi_{1}$ as

$$
\epsilon \Psi_{1}=d \Theta \chi \Psi_{0}, \quad d \Theta=-d \tau \frac{\left(v_{g}, \partial_{\tau} \Gamma_{g} \psi\right)}{\left(v_{g}, \chi \Gamma_{g} \psi\right)} .
$$

The complete asymptotic solution (to order $\epsilon$ ) becomes

$$
\Psi=\Psi_{0}+\epsilon \Psi_{1}=\Psi_{0}+d \Theta \chi \Psi_{0}
$$

One recognizes that the solution $\Psi$ represents an infinitesimal transformation of the initial wave $\Psi_{0}$ by an amount $d \Theta$. Hence, after some long time $T$ when the control parameters complete their cycle through parameter space and return to their initial values, the accumulated phase shift of the wave pattern becomes

$$
\Delta \Theta=\int d \Theta=-\int_{0}^{T} d \tau \frac{\left(v_{g}, \partial_{\tau} \Gamma_{g} \psi\right)}{\left(v_{g}, \chi \Gamma_{g} \psi\right)} .
$$

We now demonstrate that this spatial phase shift is geometrical in nature. We start by showing that this expression for $\Delta \Theta$ is in fact independent of the choice of gauge $g$. First, note that the term $\partial_{\tau} \Gamma_{g} \psi$ appearing in (5) may be expanded using the chain rule

$$
\partial_{\tau} \Gamma_{g} \psi=\frac{d g}{d \tau} \frac{d \Gamma_{g}}{d g} \psi+D \Gamma_{g}(\psi) \partial_{\tau} \psi
$$

Here, $D \Gamma_{g}(\psi)$ represents the linearization of the operator $\Gamma_{g}$ about $\psi$. The integral in (5) may therefore be decomposed into two pieces. From the identities

$$
\frac{d \Gamma_{g}}{d g} \psi=\chi \Gamma_{g} \psi=D \Gamma_{g}(\psi) \chi \psi,
$$

which follow directly from (2), one can show that the integrand of the first term constitutes a perfect differential, yielding no contribution to the phase when the parameters return to their initial values. The second term of the integral becomes

$$
\Delta \Theta=-\int_{0}^{T} d \tau \frac{\left(v_{g}, D \Gamma_{g}(\psi) \partial_{\tau} \psi\right)}{\left(v_{g}, D \Gamma_{g}(\psi) \chi \psi\right)} .
$$

Since we have assumed that the inner product is invariant under the symmetry, it follows that

$$
\left(v_{g}, \chi \Gamma_{g} \psi\right)=\left(v_{0}, \chi \psi\right), \quad \forall g,
$$

where $v_{0}$ satisfies $D \mathcal{F}(\psi)^{\dagger} v_{0}=0$. Combining this with the identities $(6)$, it follows that

$$
\Delta \Theta=-\int_{0}^{T} d \tau \frac{\left(v_{0}, \partial_{\tau} \psi\right)}{\left(v_{0}, \chi \psi\right)} .
$$

As claimed, the phase shift does not depend on choice of gauge.

We next show that the phase is also independent of time, and depends only on the path taken through parameter space. This follows trivially by writing

$$
\partial_{\tau} \psi=\nabla_{\lambda} \psi \frac{d \lambda}{d \tau}
$$

where $\nabla_{\lambda}$ denotes the derivative with respect to the parameters $\lambda$. The phase thus takes the final form

$$
\Delta \Theta=-\oint \frac{\left(v_{0}, \nabla_{\lambda} \psi\right)}{\left(v_{0}, \chi \psi\right)} d \lambda
$$

The spatial phase shift of the wave pattern, described by Eq. (9), is geometrical in that it is both independent of parametrization by time and choice of gauge $g(\lambda)$. It follows from Stokes' theorem that the phase may also be regarded as an integral of a two-form over a surface bounded by a closed path in parameter space.

As a simple illustration of these ideas, we consider a set of amplitude equations known as the Takens-Bogdanov equations with circular symmetry. These equations describe the interaction between competing instabilities in a variety of physical systems, including convection in a rotating layer [7], convection in a horizontal or vertical magnetic field [8], and lasers with a saturable absorber [9]. They take the form [10]

$$
\begin{aligned}
\frac{d z_{1}}{d t}= & z_{2}, \\
\frac{d z_{2}}{d t}= & \lambda_{1} z_{1}+\lambda_{2} z_{2}+\left(A\left|z_{1}\right|^{2}+B\left|z_{2}\right|^{2}\right) z_{1} \\
& +C\left(z_{1} \bar{z}_{2}+\bar{z}_{1} z_{2}\right) z_{1}+D\left|z_{1}\right|^{2} z_{2},
\end{aligned}
$$


where $z_{1}, z_{2}$ represent complex mode amplitudes, and $\lambda_{1}, \lambda_{2} \in \Re$ are control parameters for the system. The coefficients $A, B, C, D$ are real.

These equations are invariant under the group

$$
\operatorname{SO}(2):\left(z_{1}, z_{2}\right) \rightarrow\left(e^{i \theta} z_{1}, e^{i \theta} z_{2}\right),
$$

reflecting either the manifest circular geometry of the underlying physical system or the translational invariance of the problem accompanied by periodic boundary conditions.

Equations (10) admit a time-dependent solution of the form

$$
\left(z_{1}, z_{2}\right)=\left(R e^{i \Omega t}, i R \Omega e^{i \Omega t}\right),
$$

with

$$
R^{2}=\left(\frac{-\lambda_{2}}{D}\right), \quad \Omega^{2}=\left(\frac{\lambda_{2} A-\lambda_{1} D}{D-\lambda_{2} B}\right) .
$$

This solution corresponds to a traveling or rotating wave in the physical system. It can be shown to be stable for a certain range of parameter values. (See [10] for an extensive analysis of these equations.)

We wish to examine the behavior of this stable traveling wave when a slow time dependence is introduced into the parameters $\lambda_{1}, \lambda_{2}$. Note, however, that this solution (11) depends on the fast time $t$. We may remove this explicit time dependence by first transforming into a comoving frame via

$$
\left(z_{1}, z_{2}\right)=\left[w_{1} \exp \left(i \int_{0}^{t} \Omega d t\right), w_{2} \exp \left(i \int_{0}^{t} \Omega d t\right)\right] .
$$

The wave now appears stationary, and the preceding analysis applies. A straightforward calculation reveals

$\Delta \Theta=\oint\left[\frac{-\lambda_{2} C+A+B \Omega^{2}}{2 \lambda_{2} \Omega\left(\lambda_{2} B-D\right)}\right] d \lambda_{2}+\left[\frac{A+B \Omega^{2}}{2 \Omega^{2}\left(\lambda_{2} B-D\right)}\right] d \Omega$

(For simplicity, we have expressed this phase as a line integral involving $\lambda_{2}, \Omega$ instead of $\lambda_{1}, \lambda_{2}$.)

In this example, note that the term $\int_{0}^{t} \Omega d t$, obtained when transforming into a moving reference frame, may be regarded as a "dynamical" phase for the system. This dynamical phase is entirely analogous to that found in quantum systems during adiabatic transport of parameters. In general, such dynamical phases arise in dissipative systems when nonstationary waves are studied. The simple trick used here of transforming to a comoving frame shows how various propagating waves can be studied within the same general framework as stationary waves. This procedure can in fact be formalized, and a more complete description will appear elsewhere [11].

Last, we wish to point out a connection between the spatial phase shift discussed here and the temporal phase addressed in [5]. Although we have dealt only with spatial symmetries of a system, the temporal geometric phase may now be seen as corresponding to the special case of time-translation symmetry. In particular, adiabatic transport induces motion along the direction defined by the group action (time translation), thereby producing a temporal shift. For the particular example given above, these two notions in fact coincide, since the action of the rotation group $\mathrm{SO}(2)$ on the solution (11) is identical to that of time translation.

In summary, we have shown that a geometrical phase analogous to that found by Berry presents itself in the non-Hamiltonian setting as well. The phase is associated with motion along the neutral mode of the system, as defined by the continuous symmetry. Dissipation plays a prominent role in this scenario by providing for a rapid collapse of the system onto the stable solution during adiabatic transport. The phase shift discussed here, associated with the spatial symmetry of the problem, complements the recent discovery [5] of a temporal phase shift in dissipative systems. The geometrical phase should be readily detectable through experiment, and an examination of wave patterns in fluids and other continuous media should provide an interesting realization of this phenomenon.

The author thanks Michael Crescimanno and Eric Friedman for their valuable insights and suggestions, and gratefully acknowledges useful discussions with Gregory Keaton, Edgar Knobloch, and Robert Littlejohn.

[1] M. V. Berry, Proc. R. Soc. London A 392, 45 (1984)

[2] J. H. Hannay, J. Phys. A 18, 221 (1985).

[3] Y. Aharonov and J. Anandan, Phys. Rev. Lett. 58, 1593 (1987).

[4] J. Garrison and R. Chiao, Phys. Rev. Lett. 60, 165 (1988).

[5] T. B. Kepler and M. L. Kagan, Phys. Rev. Lett. 66, 847 (1991).

[6] M. Golubitsky, I. Stewart, and D. G. Schaeffer, Singularities and Groups in Bifurcation Theory (Springer-Verlag, New York, 1988), Vol. II, p. 31.

[7] E. Knobloch and M. Silber, Geophys. Astrophys. Fluid Dyn. 51, 195 (1990).

[8] G. Dangelmayr and E. Knobloch, Phys. Lett. A 117, 394 (1986).

[9] G. Dangelmayr and M. Neveling, J. Phys. A 22, 1291 (1989).

[10] G. Dangelmayr and E. Knobloch, Philos. Trans. R. Soc. A 332, 243 (1987).

[11] A. S. Landsberg (to be published). 